\title{
Islands and Page curves in charged dilaton black holes
}

\author{
Ming-Hui Yu ${ }^{1}$, Xian-Hui Ge $e^{1,2, a}$ \\ ${ }^{1}$ Department of Physics, Shanghai University, Shanghai 200444, China \\ ${ }^{2}$ Center for Gravitation and Cosmology, College of Physical Science and Technology, Yangzhou University, Yangzhou 225009, China
}

Received: 16 September 2021 / Accepted: 9 December 2021 / Published online: 8 January 2022

(C) The Author(s) 2022

\begin{abstract}
We study the Page curve for eternal GarfinkleHorowitz-Strominger dilaton black holes in four dimensional asymptotically flat spacetime by using the island paradigm. The results demonstrate that without the island, the entanglement entropy of Hawking radiation is proportional to time and becomes divergent at late times. While taking account of the existence of the island outside the event horizon, the entanglement entropy stops growing at late times and eventually reaches a saturation value. This value is twice of the Bekenstein-Hawking entropy and consistent with the finiteness of the von Neumann entropy of eternal black holes. Moreover, we discuss the impact of the stringy coefficient $n$ and charge $Q$ on the Page time and the scrambling time respectively. For the non-extremal case, the influence of the coefficient $n$ on them is small compared to the influence of the charge $Q$. However, for the extremal case, the Page time and the scrambling time become divergent or near vanishing. This implies the island paradigm needs further investigation.
\end{abstract}

\section{Contents}

1 Introduction ................ 1

2 Charged dilaton black holes . . . . . . . . . . 3

3 Entanglement entropy without island is divergent . . 4

4 Entanglement entropy in the presence of island ... 5

4.1 Island absent at early times . . . . . . . . . . 5 5

4.2 Island emerged at late times . . . . . . . . . . 6

5 Page time and scrambling time . . . . . . . . 8

6 Conclusion and discussion . . . . . . . . . . . 10

Note added . . . . . . . . . . . . . . . . . 11

References . . . . . . . . . . . . . . 12

a e-mail: gexh@shu.edu.cn (corresponding author)

\section{Introduction}

The black hole information paradox [1] is the fundamental problem of most significant fields-quantum mechanics and general relativity-in theoretical physics, and has long been regarded as the key to the study of quantum gravity. Recently, there has been a breakthrough in the calculation for the finegrained entropy of radiation of black holes by using the island paradigm [2-6]. The island paradigm can not only obtain the Page curve from the perspective of gravity, but also explain the attribution of the degree of freedom of the black hole interior.

The black hole information paradox is first initiated by Hawking in 1975. The outgoing Hawking radiation is proved to be a mixed (thermal) state obeying the Planck law (neglecting the graybody factor) and this result does not depend on the initial condition [7]. However, this result is contrary to the basic assumption of quantum mechanics - the unitarity principle, because the evaporating process evolves from a pure state to a mixed state and information that falls into the black hole will disappear forever after the black hole evaporated. If we assume that a black hole evaporates from a pure quantum state, based on the principle of unitarity, the radiation must still be in a pure quantum state instead of a mixed state at the end of evaporation. A resolution of the black hole information paradox is proposed by Page: if the unitary evolution does not change the von Neumann entropy (the fine-grained entropy) of the system, the time evolution of the fine-grained entanglement entropy of Hawking radiation in this process is described by the Page curve [8,9]. Accordingly, whether the black hole information issue can be solved is converted into whether the Page curve of the evaporating black hole can be reproduced.

However, for a long time afterwards, physicists have been inconclusive on whether the evaporation process of a black hole satisfies the unitarity principle, until the discovery of the AdS/CFT duality [10]. This theory provides a strong physical indication that the system in anti-de Sitter spacetime 
(AdS) corresponds to the conformal field theory (CFT) on the boundary. That is to say, the evaporation process of black holes can be described by the boundary CFT, in which CFT is based on quantum mechanics and satisfies the principle of unitarity. Therefore, for the evaporation of black holes, the fine-grained entropy of Hawking radiation should obey the Page curve.

Until recent years, the task to reproduce the Page curve is still difficult, because according to the consideration of quantum theory, the event horizon is transformed into a high energy area - the "firewall", which is also hostile to the classical general relativity [11]. As the black hole evaporates, the amount of radiation entropy increases endlessly at late times. In the case of eternal black holes, the amount of radiation approaches infinity and leads to infinite amount of entropy, which obviously violates the principle of unitarity. However, unitarity limits the maximum entropy of a black hole, which cannot exceed the Bekenstein-Hawking entropy (the coarsegrained entropy) [12]. Therefore, the eternal black hole information paradox can be solved by checking whether its Page curve reaches a bounded value, i.e. the Bekenstein-Hawking entropy.

Up to now, the calculation of the Page curve is initially implemented by using the semiclassical theory of the JT (Jackiw-Teitelboim) gravity in the context of asymptotically AdS spacetime $[2,4,13]$. Compared to higher dimensional systems, the structure of two-dimensional systems can provide more tractable analyses. Therefore, most of the current study is conducted in the two-dimensional gravity system. These studies indicates that islands emerge at late times. Under different backgrounds of spacetime, the location of islands could be outside or inside the event horizon [1418]. The effect of islands maintains the finiteness of the vonNeumann entropy of the black hole Hawking radiation. At late times, the entanglement entropy takes the BekensteinHawking entropy for eternal black holes. Due to the complexity of the higher dimensional spacetime structure, the corresponding Page curve is difficult to calculate, so there are less work in this situation. However, related study suggests that the island is connected to the external radiation through wormholes in higher dimensional spacetime [3]. Now, there are already many interesting and meaningful works [19-31], especially Page curves and islands in Schwarzschild black holes and Reissner-Nordström black holes $[19,20]$.

One of the key applications of the AdS/CFT duality is the holographic calculation of the entanglement entropy in quantum field theory, which is the famous holographic entanglement entropy conjecture proposed by Ryu and Takayanagi (RT) [32]. This conjecture transforms the calculation of the entanglement entropy in quantum field theory to a minimal surface in AdS spacetime. In the subsequent papers, after considering quantum corrections [33-35], the RT proposal is extended to the prescription of "Quantum Extremal Sur- face" (QES) [36]. Up using this prescription, the results show that islands emerge at late times, causing the entanglement entropy of the radiation finite. Moreover, in the presence of the island, the behavior of the entanglement entropy obeys the Page curve. Accordingly, the island formula for the finegrained entropy of the Hawking radiation is further derived by $[37,38]$ :

$S(R)=\min \left\{\operatorname{ext}\left[\frac{\operatorname{Area}(\partial I)}{4 G_{N}}+S_{\text {matter }}(R \cup I)\right]\right\}$,

where $G_{N}$ denotes the Newton constant, $R$ is the radiation and $I$ is the island, $\partial I$ is the boundary of the island, and $S_{\text {matter }}$ denotes the von Neumann entropy contributed by the quantum matter field around the black hole. Equivalently, the island formula (1.1) can be derived mathematically by using the replica trick for gravitational theories $[2,39]$.

Now, let us explain in detail the physical meaning of the island formula (1.1). As mentioned above, the entanglement entropy after quantum corrections is considered in the prescription of "QES", which is called the generalized entropy. It not only receives contribution from the surface term of the island but also the von Neumann entropy of the combination of the union of the radiation region $R$ and the island region $I$, which is written as

$S_{\text {gen }}=\frac{\operatorname{Area}(\partial I)}{4 G_{N}}+S_{\text {matter }}(R \cup I)$.

The fine-grained entropy of the Hawking radiation of the system is found by evaluating generalized entropy value under all extremal points and pick the minimal value. These extremal points corresponds to the location of islands. No extremal points mean no island. We will see in later sections that it is the important interaction of two contributions that causes a phase transition of the entanglement entropy at the Page time. Accordingly, the entanglement entropy of the radiation will be constrained by the unitarity after the Page time.

Since the most promising approach realizing the unified theories is the string theory at present, which can be reduced to the Einstein-Maxwell dilaton gravity in the low-energy limit. Therefore, it is meaningful to study the charged dilaton gravity model. In this paper, we consider the two-sided geometry for quantum systems coupled with gravity. Different from the evaporation in the AdS spacetime, where a coupling auxiliary system is needed to absorb the Hawking radiation, we work in the asymptotically flat spacetime, so that the Hawking quanta is created near the horizon and naturally launched to infinity. We calculate the entanglement entropy for asymptotically flat eternal Garfinkle-HorowitzStrominger dilaton (GHS) black holes and construct the corresponding Page curve. The result show that the entanglement entropy of Hawking radiation converges at late times in the presence of the island, satisfying the finiteness of the 
von Neumann entropy of the finite system. In addition, we also study the influence of the stringy coefficient $n$ and charge $Q$ on the Page time and the scrambling time.

The content of the paper is organized as follows. In Sect. 2, we briefly introduce properties of charged dilaton black holes. In Sect. 3, we calculate the entanglement entropy in the construction without island and reveal the information paradox for GHS black holes. In Sect. 4, we analyze the behavior of the generalized entropy at early times and late times. Once we consider the construction of a single island, the unitary result is obtained. In Sect. 5, based on the previous result, we discussed the relationship between the Page time and the scrambling to the stringy coefficient $n$ and the charge $Q$. Conclusion and discussion are given in the last section.

\section{Charged dilaton black holes}

In this section, we review basic properties of GarfinkleHorowitz-Strominger dilaton black holes, which is a family representing static, spherically symmetric charged black holes in low-energy string theory [40,41]. Different from the Reissner-Nordström (RN) black hole in the classical general relativity, they are labeled by asymptotic value of the scalar dilaton, which can produce some interesting results.

The gravitational part of the action is generalized to the following form

$I=\int d^{4} x \sqrt{-g}\left[R-2 g^{\mu \nu} \nabla_{\mu} \varphi \nabla_{\nu} \varphi+e^{-2 \alpha \varphi} F_{\mu \nu} F^{\mu \nu}\right]$,

where $R$ determines the Ricci scalar curvature, $\varphi$ denotes the dilaton, $F$ is the Maxwell tensor, and the exponent $\alpha$ represents the coupling between the electromagnetic field and the dilaton field.

The metric of a static, spherically symmetric charged dilaton black hole can be written as follows

$d s^{2}=-f(r) d t^{2}+f^{-1}(r) d r^{2}+R^{2}(r)\left(d \theta^{2}+\sin ^{2} \theta d \phi^{2}\right)$,

with the metric function $f(r)$ is defined by

$f(r)=\left(1-\frac{r_{+}}{r}\right)\left(1-\frac{r_{-}}{r}\right)^{n}$,

and

$R^{2}(r)=r^{2}\left(1-\frac{r_{-}}{r}\right)^{1-n}$.

The associated dilaton field and the potential are

$e^{2 \alpha \varphi}=R^{2}(r)=r^{2}\left(1-\frac{r_{-}}{r}\right)^{1-n}$,
$A=\frac{Q}{r} d t$,

$F=d A=-\frac{Q}{r^{2}} d t \wedge d r$.

In these equations, $n$ is the stringy coefficient has been introduced for convenience.

$n=\frac{1-\alpha^{2}}{1+\alpha^{2}}$,

with $\alpha \in[0,1]$, in particular, when $\alpha=0, \varphi=0$, so $n$ monotonically varies in the interval $[0,1]$. In these equations, the Planck units are used $\hbar=G_{N}=c=k=1$ hereafter. The constant $r_{+}$is the outer event horizon. Note that, $r_{-}$is not an inner horizon but a spacelike singularity for any non-vanishing $\alpha$ [40]. This is an important nature generated by dilation fields. The constants $r_{ \pm}$can be expressed respectively as follows

$r_{+}=M\left[1+\sqrt{1-\frac{2 n}{1+n}\left(\frac{Q}{M}\right)^{2}}\right]$,

$r_{-}=\frac{M}{n}\left[1-\sqrt{1-\frac{2 n}{1+n}\left(\frac{Q}{M}\right)^{2}}\right]$,

where $M$ is the mass and $Q$ is the charge in terms of $r_{+}$and $r_{-}$

$M=\frac{1}{2} r_{+}+\frac{n}{2} r_{-}, \quad Q^{2}=\frac{(1+n)}{2} r_{+} r_{-}$.

Notice that the two constants $r_{+}$and $r_{-}$coincide at

$\frac{Q}{M}=\sqrt{\frac{2}{1+n}}$,

which corresponds to the extremal charged dilation black hole. The surface gravity of the event horizon is

$\kappa_{+}=\frac{1}{2 r_{+}}\left(1-\frac{r_{-}}{r_{+}}\right)^{n}$.

Thus the Hawking temperature is

$T_{+}=\frac{\kappa_{+}}{2 \pi}=\frac{1}{4 \pi r_{+}}\left(1-\frac{r_{-}}{r_{+}}\right)^{n}$.

The area of the event horizon is

$A_{+}=4 \pi R^{2}\left(r_{+}\right)=4 \pi r_{+}^{2}\left(1-\frac{r_{-}}{r_{+}}\right)^{1-n}$,

and the Bekenstein-Hawking entropy of black hole read as

$S_{B H}=\frac{A_{+}}{4 G_{N}}=\frac{\pi r_{+}^{2}}{G_{N}}\left(1-\frac{r_{-}}{r_{+}}\right)^{1-n}$.

Interestingly, the area of the event horizon and the black hole entropy becomes zero in the extremal limit $\left(r_{+}=r_{-}\right)$. We also notice that in the limit $n=1$, the metric restores the Reissner-Nordström (RN) black hole, while $n=0$, 
the metric corresponds to the Gibbons-Maeda-GarfinkleHorowitz-Strominger (GMGHS) black hole. When the charge $Q=0$, the Schwarzschild black hole with fixed event horizon $r_{+}=2 M$ is recovered.

\section{Entanglement entropy without island is divergent}

In this section, we calculate the entanglement entropy of the Hawking radiation in the absence of the island and check GHS black holes information paradox more intuitively at late times. Notice that without island, the first term of the generalized entropy (1.2) is vanishing. Accordingly, there is only the contribution from the radiation in the second term. Therefore, we have only two points on the cut-off surface, which corresponds to boundaries of radiation regions at the left wedge $R_{-}$and the right wedge $R_{+}$(see Fig. 1).

In four or higher dimensional spacetime, the expression of the entanglement entropy is very complicated. However, in the case of low energy, the expression can be well approximated by the theory of two-dimensional quantum field. For an observer located at the asymptotic infinity, the Hawking radiation can be described by the two-dimensional $s$-wave without angular momentum [16,17]. Apart from that, we assume that the system in the pure quantum state at the initial time. In this case, according to the complementarity of the von-Neumann entropy, the entanglement entropy of the radiation region is equal to the entanglement entropy of the region $\left[b_{-}, b_{+}\right]$, which can be written as [42]

$S_{R}($ without island $)=S_{\text {matter }}(R)=\frac{c}{3} \log \left[d\left(b_{+}, b_{-}\right)\right]$,

where $c$ is the central charge in CFT, and $d\left(b_{+}, b_{-}\right)$denotes the distance between the points $b_{+}$and $b_{-}$in the GHS geometry. Here, we set spacetime coordinates for them as $b_{ \pm}=(t, r)=\left( \pm t_{b}, b\right)$. On the other hand, we find that it is useful to employ the Kruskal transformation for the metric (2.2), which can smoothly cover the maximum extension of the two-side geometry. Defining the Kruskal coordinate as

$U=-e^{-\kappa_{+}\left(t-r^{*}\right)}, \quad V=e^{\kappa_{+}\left(t+r^{*}\right)}$,

with the tortoise coordinate $r^{*}=\int \frac{1}{f(r)} d r$, we are interested in the near horizon region where $r \sim r_{+}$, thus

$$
\begin{aligned}
r^{*}(r) & \simeq\left(\frac{r_{+}}{r_{+}-r_{-}}\right)^{n}\left[r+r_{+} \log \frac{r-r_{+}}{r_{+}}\right] \\
& =\frac{1}{2 \kappa_{+} r_{+}}\left[r+r_{+} \log \frac{r-r_{+}}{r_{+}}\right] .
\end{aligned}
$$

Under the Kruskal transformation, the metric (2.2) becomes the following form

$d s^{2}=-g^{2}(r) d U d V+R^{2}(r) d \Omega^{2}$, where $d \Omega^{2}=d \theta^{2}+\sin ^{2} \theta d \phi^{2}$. The function $g^{2}(r)$ is called the conformal factor, which can be written as follows

$g^{2}(r)=\frac{f(r)}{\kappa_{+}^{2} e^{2 \kappa_{+} r^{*}(r)}}$.

It is regular at the event horizon

$$
\begin{aligned}
\lim _{r \rightarrow r_{+}} g^{2}(r) & =\lim _{r \rightarrow r_{+}} \frac{\left(1-\frac{r_{+}}{r}\right)\left(1-\frac{r_{+}}{r}\right)^{n}}{\kappa_{+}^{2} \exp \left[\frac{r}{r_{+}}+\log \frac{r-r_{+}}{r_{+}}\right]} \\
& =\left(1-\frac{r_{-}}{r_{+}}\right)^{n} \kappa_{+}^{-2} .
\end{aligned}
$$

According to the conformal mapping, the matter sector of the radiation entropy in the GHS geometry contributes as

$$
\begin{aligned}
& S_{\text {matter }}(R) \\
& \quad=\frac{c}{6} \log \left\{g\left(b_{-}\right) g\left(b_{+}\right)\left[U\left(b_{-}\right)-U\left(b_{+}\right)\right]\right. \\
& \left.\left[V\left(b_{+}\right)-V\left(b_{-}\right)\right]\right\},
\end{aligned}
$$

where

$\sqrt{g(x) g(y)[U(y)-U(x)][V(x)-V(y)]}=d(x, y)$,

denotes the geodesic distance between the points $x$ and $y$ in the GHS geometry. Substituting coordinates of points $b_{ \pm}$, we can obtain ${ }^{1}$

$$
\begin{aligned}
S_{\text {matter }}(R)= & \frac{c}{6}\left[\frac{4 f(b)}{\kappa_{+}^{2}} \cosh ^{2}\left(\kappa_{+} t_{b}\right)\right] \\
= & \frac{c}{6} \log \left[16 r_{+}^{2}\left(1-\frac{r_{-}}{r_{+}}\right)^{-2 n}\left(1-\frac{r_{+}}{b}\right)\right. \\
& \left.\times\left(1-\frac{r_{-}}{b}\right)^{n} \cosh ^{2}\left(\kappa_{+} t_{b}\right)\right] .
\end{aligned}
$$

At late times, we assume that $t_{b} \gg b\left(>r_{+}\right)$, so we can employ the approximation

$\cosh \left(\kappa_{+} t_{b}\right) \simeq \frac{1}{2} e^{\kappa+t_{b}}$.

The above result can be recast as

$$
\begin{aligned}
S_{\text {matter }}(R) & \sim \frac{c}{3} \log \left[\cosh \left(\kappa_{+} t_{b}\right)\right] \\
& \simeq \frac{c}{3} \kappa_{+} t_{b} .
\end{aligned}
$$

Obviously, the entropy increases linearly with time and becomes infinite at late times (see Fig. 3). Therefore, there is no Page curve in the absence of island. The entanglement entropy is ultimately infinite and larger than the BekensteinHawking entropy of the black hole. This result contradicts

\footnotetext{
${ }^{1}$ We should be cautiously swapping the signs of $U$ and $V$ in (3.2) when considering points in the left region in the Fig. 1, since these points correspond to $r<0$.
} 
Fig. 1 Penrose diagram for GHS black holes without island. The left for $0 \leq n<1$, while the right for $n=1$. The Hawking radiation is emitted from the cut-off surface that denoted as $b_{ \pm}$
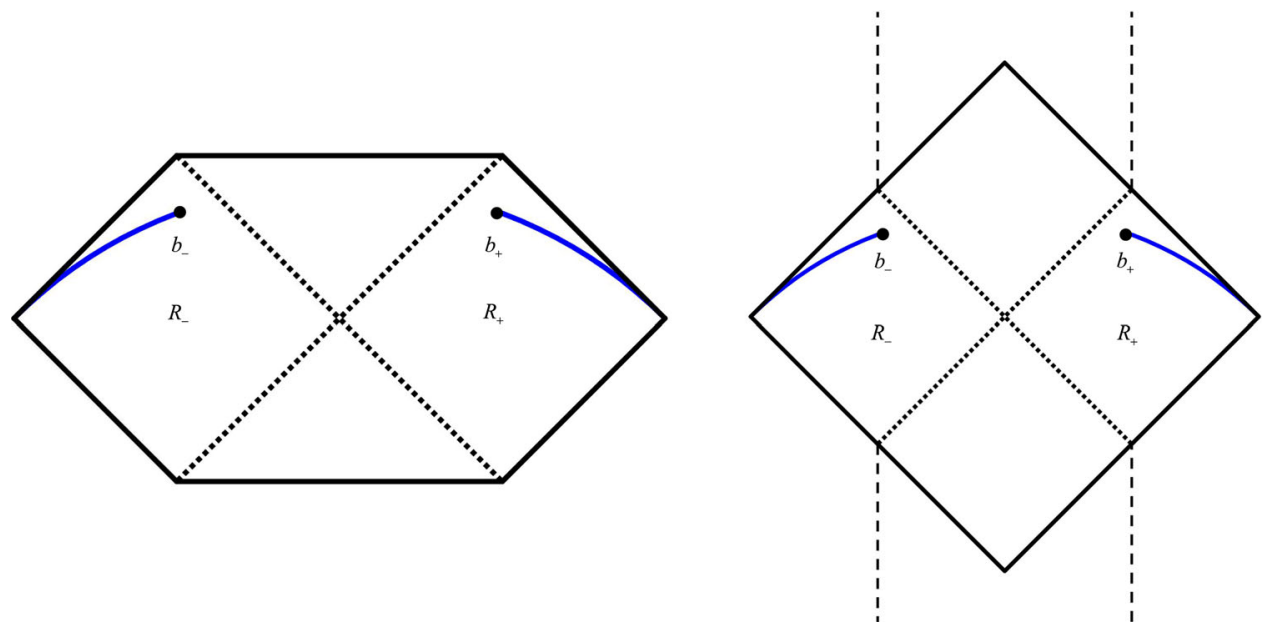

our previous assumptions. For the unitary evolution of eternal black holes, the entanglement entropy is at most twice of the Bekenstein-Hawking entropy at late times. Therefore, the paradox appears. In next section, we calculate the entanglement entropy in the construction with an island and as long as this construction is considered, the unitary Page curve is reproduced.

\section{Entanglement entropy in the presence of island}

In this section, we calculate the entanglement entropy by considering the island. The construction is shown in Fig. 2. Thus, we should consider the contribution of the island in the second term of the generalized entropy (1.2).

The boundary of the island is set as $a_{ \pm}=(t, r)=$ $\left( \pm t_{a}, a\right)$. For this construction, the contribution from entropy of the matter field depends on the distance between the cutoff surface $b_{ \pm}$and the event horizon $r_{+}$, but we only focus on the behavior of the entanglement entropy at late times. Therefore, we assume that the cut-off surface is far away from the event horizon, $b \gg r_{+}$. Besides, we still take the $s$ wave approximation. Under this approximation, the formula of the entanglement entropy in the two disconnected intervals of the radiation region $R$ and the island region $I$ can be written as follows [43]

$$
\begin{aligned}
& S_{R}(\text { with island }) \\
& \quad=S_{\text {matter }}(R \cup I) \\
& \quad=\frac{c}{3} \log \left[\frac{d\left(a_{+}, a_{-}\right) d\left(b_{+}, b_{-}\right) d\left(a_{+}, b_{+}\right) d\left(a_{-}, b_{-}\right)}{d\left(a_{+}, b_{-}\right) d\left(a_{-}, b_{+}\right)}\right] .
\end{aligned}
$$

Accordingly, the generalized entropy (1.2) in this construction read as

$$
S_{\text {gen }}=\frac{2 \pi R^{2}(a)}{G_{N}}+S_{\text {matter }}(R \cup I),
$$

where $R^{2}(a)$ is the area of the island. By using (3.8), the explicit expression is obtained by

$$
\begin{aligned}
S_{\text {gen }}= & \frac{2 \pi R^{2}(a)}{G_{N}}+\frac{c}{6} \log \left\{2^{8} r_{+}^{4}\left(1-\frac{r_{-}}{r_{+}}\right)^{-4 n}\left(1-\frac{r_{+}}{a}\right)\right. \\
& \times\left(1-\frac{r_{+}}{b}\right)\left[\left(1-\frac{r_{-}}{a}\right)\left(1-\frac{r_{-}}{b}\right)\right]^{n} \\
& \left.\cosh ^{2}\left(\kappa_{+} t_{a}\right) \cosh ^{2}\left(\kappa_{+} t_{b}\right)\right\} \\
& +\frac{c}{3} \log \frac{\cosh \left[\kappa_{+}\left(r^{*}(a)-r^{*}(b)\right)\right]-\cosh \left[\kappa_{+}\left(t_{a}-t_{b}\right)\right]}{\cosh \left[\kappa_{+}\left(r^{*}(a)-r^{*}(b)\right)\right]+\cosh \left[\kappa_{+}\left(t_{a}+t_{b}\right)\right]} .
\end{aligned}
$$

Here $r^{*}(a, b)$ is the tortoise coordinate, which is defined in (3.3). After extremizing the above expression, the finegrained entropy of the radiation is obtained by minimizing the generalized entropy. In next subsections, we can find that the island emerges at late times, which make the entropy stops increasing.

\subsection{Island absent at early times}

Firstly, we study the behavior of the entanglement entropy at early times. Since (4.3) is too complicated, some appropriate approximations should be taken. At early times, we assume that $t_{a}, t_{b} \ll r_{+}$and pick up the cut-off surface far away from the event horizon $\left(b \gg r_{+}\right)$. Therefore, the last term of $S_{\text {gen }}$ (4.3) can be ignored

$$
\frac{c}{3} \log \frac{\cosh \left[\kappa_{+}\left(r^{*}(a)-r^{*}(b)\right)\right]-\cosh \left[\kappa_{+}\left(t_{a}-t_{b}\right)\right]}{\cosh \left[\kappa_{+}\left(r^{*}(a)-r^{*}(b)\right)\right]+\cosh \left[\kappa_{+}\left(t_{a}+t_{b}\right)\right]} \rightarrow 0,
$$

and we have

$$
\left(1-\frac{r_{+}}{b}\right)\left(1-\frac{r_{-}}{b}\right)^{n} \simeq 1, \quad \cosh ^{2}\left[\kappa_{+}\left(t_{a}, t_{b}\right)\right] \simeq 1 .
$$


Fig. 2 Penrose diagram for GHS black holes with the island. The left for $0 \leq n<1$, while the right for $n=1$. The boundary of the island is located at $a_{ \pm}$. The cutoff surface is denoted as $b_{ \pm}$. Notice that the location of island where extends to outside the event horizon

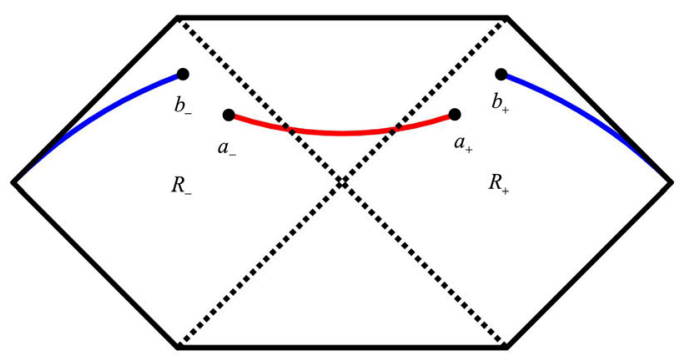

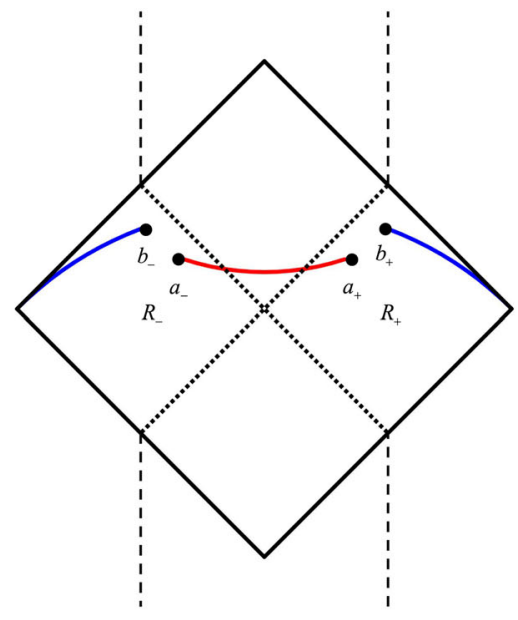

4.2 Island emerged at late times

Now, we focus on the behavior of the entropy at late times. At the end stage of evaporation, the island locates at the region of black hole. However, the region inside the island belongs to the external radiation region. As a result, the early Hawking radiation is actually the internal partner inside the black hole. Considering the two together and one obtain a pure state. Therefore, the growth of the entanglement entropy is stop. The behavior of the entropy is constrained by unitarity as we expect.

For this construction at late times, we assume that $t_{a}, t_{b} \gg$ $b\left(>r_{+}\right)$. We first analysis the time dependence of (4.3)

$$
\begin{aligned}
& S_{\mathrm{gen}}(t)=\frac{c}{3} \log \left\{\cosh \left(\kappa_{+} t_{a}\right) \cosh \left(\kappa_{+} t_{b}\right)\right. \\
& \left.\frac{\cosh \left[\kappa_{+}\left(r^{*}(a)-r^{*}(b)\right)\right]-\cosh \left[\kappa_{+}\left(t_{a}-t_{b}\right)\right]}{\cosh \left[\kappa_{+}\left(r^{*}(a)-r^{*}(b)\right)\right]+\cosh \left[\kappa_{+}\left(t_{a}+t_{b}\right)\right]}\right\} .
\end{aligned}
$$

Employing the following approximations

$\cosh \kappa_{+} t_{a} \simeq \frac{1}{2} e^{\kappa_{+} t_{a}}, \quad \cosh \kappa_{+} t_{b} \simeq \frac{1}{2} e^{\kappa_{+} t_{b}}$,

and

$$
\cosh \left[\kappa_{+}\left(t_{a}+t_{b}\right)\right] \gg \cosh \left[\kappa_{+}\left(r^{*}(a)-r^{*}(b)\right)\right],
$$

the expression (4.9) is reduced as

$$
\begin{aligned}
& S_{\text {gen }}(t) \simeq \frac{c}{3} \log \left\{\cosh \left[\kappa_{+}\left(r^{*}(a)-r^{*}(b)\right)\right]\right. \\
& \left.-\cosh \left[\kappa_{+}\left(t_{a}-t_{b}\right)\right]\right\} .
\end{aligned}
$$

We can easily find that when $\cosh \left[\kappa_{+}\left(t_{a}-t_{b}\right)\right]$ to reach the minimum, i.e. $t_{a}=t_{b}$, the expression obtains the maximal value. Setting $t_{a}=t_{b}=t$ and substituting into the expression, we obtain the expression that does not depend on time. This implies that the entanglement entropy converges at late times.

Next, we calculate the explicit form for the generalized entropy (4.3) at late times. Invoking all of approximations 
(4.10) and (4.11). We also approximate

$\cosh \left[\kappa_{+}\left(r^{*}(a)-r^{*}(b)\right)\right] \simeq \frac{1}{2} e^{\kappa_{+}\left[r^{*}(b)-r^{*}(a)\right]}$.

Through the above approximations, the generalized entropy (4.3) can be recast as

$$
\begin{aligned}
& S_{\text {gen }}^{(\text {late })}=\frac{2 \pi R^{2}(a)}{G_{N}}+\frac{c}{6} \log \left\{2^{8} r_{+}^{4}\left(1-\frac{r_{-}}{r_{+}}\right)^{-4 n}\left(1-\frac{r_{+}}{a}\right)\right. \\
& \times\left(1-\frac{r_{+}}{b}\right)\left[\left(1-\frac{r_{-}}{a}\right)\left(1-\frac{r_{-}}{b}\right)\right]^{n} \\
& \left.\times \cosh ^{2} \kappa_{+} t_{a} \cosh ^{2} \kappa_{+} t_{b}\right\} \\
& +\frac{c}{3} \log \frac{\cosh \left[\kappa_{+}\left(r^{*}(a)-r^{*}(b)\right)\right]-\cosh \left[\kappa_{+}\left(t_{a}-t_{b}\right)\right]}{\cosh \left[\kappa_{+}\left(r^{*}(a)-r^{*}(b)\right)\right]+\cosh \left[\kappa_{+}\left(t_{a}+t_{b}\right)\right]} \\
& \simeq \frac{2 \pi R^{2}(a)}{G_{N}}+\frac{c}{6} \log \left[\frac{16 f(a) f(b)}{\kappa_{+}^{4}} \cosh ^{4} \kappa_{+} t\right] \\
& +\frac{c}{3} \log \frac{\frac{1}{2} e^{\kappa_{+}\left[r^{*}(b)-r^{*}(a)\right]}-1}{\frac{1}{2} e^{\kappa+\left[r^{*}(b)-r^{*}(a)\right]}+\frac{1}{2} e^{2 \kappa+t}} \\
& \simeq \frac{2 \pi R^{2}(a)}{G_{N}} \\
& +\frac{c}{3} \log \left[4 g(a) g(b) e^{\kappa_{+}\left(r^{*}(a)+r^{*}(b)\right)} \cosh ^{2} \kappa_{+} t\right] \\
& +\frac{c}{3} \log \frac{\left[e^{\kappa_{+}\left[r^{*}(b)-r^{*}(a)\right]}-2\right] e^{-2 \kappa+t}}{e^{\kappa_{+}\left[r^{*}(b)-r^{*}(a)-2 t\right]}+1} \\
& =\frac{2 \pi R^{2}(a)}{G_{N}}+\frac{c}{3} \log \frac{1-2 e^{\kappa_{+}\left[r^{*}(a)-r^{*}(b)\right]}}{1+e^{\kappa_{+}\left[r^{*}(b)-r^{*}(a)-2 t\right]}} \\
& +\frac{2 c}{3} \kappa_{+} r^{*}(b)+\frac{c}{3} \log [g(a) g(b)] \\
& =\frac{2 \pi R^{2}(a)}{G_{N}}+\frac{2 c}{3} \kappa_{+} r^{*}(b)+\frac{c}{3} \log [g(a) g(b)] \\
& +\frac{c}{3} \log \left[1+\frac{-2 e^{\kappa_{+}\left(r^{*}(a)-r^{*}(b)\right)}-e^{\kappa_{+}\left[r^{*}(b)-r^{*}(a)-2 t\right]}}{1+e^{\kappa_{+}\left[r^{*}(b)-r^{*}(a)-2 t\right]}}\right] \\
& \simeq \frac{2 \pi R^{2}(a)}{G_{N}}+\frac{2 c}{3} \kappa_{+} r^{*}(b)+\frac{c}{3} \log [g(a) g(b)] \\
& -\frac{2 c}{3} e^{\kappa_{+}\left[r^{*}(a)-r^{*}(b)\right]}-\frac{c}{3} e^{\kappa_{+}\left[r^{*}(b)-r^{*}(a)-2 t\right]} .
\end{aligned}
$$

In the first approximately equal sign, we use (4.13) and set $t_{a}=t_{b}=t$. In the second approximately equal sign, we use the conformal factor (3.5) to simplify. In the last line, we apply the first order expansion of the logarithm, $\lim _{x \rightarrow 0} \log (1+x) \simeq x$.

Notice that the last term in the expression has dependence on the time $t$. However, it is the subleading order and quickly decays with time. Therefore, at late times, we can guarantee that the location of island is a constant that does not depend on time. Our current goal is to determine the location of the island. Extremizing this expression with respect to $a$

$$
\begin{aligned}
\frac{\partial S_{\mathrm{gen}}^{(\text {late })}}{\partial a}= & \frac{2 \pi}{G_{N}}\left(1-\frac{r_{-}}{a}\right)^{-n}\left[2 a-(n+1) r_{-}\right]+\frac{c}{3} \frac{g^{\prime}(a)}{g(a)} \\
& -\frac{2 c \kappa_{+}}{3 f(a)} e^{\kappa+\left[r^{*}(a)-r^{*}(b)\right]} \\
= & \frac{2 \pi}{G_{N}}\left(\frac{a}{a-r_{-}}\right)^{n}\left[2 a-(n+1) r_{-}\right] \\
& +\frac{c}{6}\left[\frac{f^{\prime}(a)-2 \kappa_{+}}{f(a)}\right] \\
& -\frac{2 c \kappa_{+}}{3 f(a)} e^{\kappa+\left[r^{*}(a)-r^{*}(b)\right]} .
\end{aligned}
$$

By using $t_{a}=t_{b}=t$ and $a \sim r_{+}$, we obtain the location of the island as follows

$$
\begin{aligned}
a & \simeq r_{+}+\frac{c G_{N}}{3 \pi}\left[\frac{\kappa_{+} r_{+}}{2 r_{+}-(n+1) r_{-}}\right] e^{\kappa_{+}\left[r_{+}-r^{*}(b)\right]}+\mathcal{O}\left(G_{N}^{2}\right) \\
& \simeq r_{+}+\mathcal{O}\left(\frac{c G_{N}}{r_{+}}\right)
\end{aligned}
$$

We can clearly see that the boundary of the island locates outside the event horizon, which is consistent with Fig. 2. Substituting this location into (4.3). Finally, the fine-grained entropy of Hawking radiation at late times is obtained as

$$
\begin{aligned}
S_{E E}= & \frac{2 \pi r_{+}^{2}}{G_{N}}\left(1-\frac{r_{-}}{r_{+}}\right)^{1-n} \\
& +\frac{c}{3} \log \left[\frac{1}{\kappa_{+}^{2}}\left(\frac{b-r_{+}}{b}\right)\left(\frac{r_{+}-r_{-}}{r_{+}}\right)^{n} e^{2 \kappa_{+} b}\right]+\cdots \\
\simeq & 2 S_{B H} .
\end{aligned}
$$

The leading order of this expression is the BekensteinHawking entropy of the black hole (2.13), which is produced by considering the construction of the island. The subleading order and higher order terms are negligible compared to $S_{B H}$.

Based on these results, we briefly discuss the behavior of the generalized entropy. At early times, there is no island. The generalized entropy is dominated by the contribution from the matter part and grows with time. At late times, we consider the construction of the island that locates outside the event horizon. The emergence of the island makes the degree of freedom of the black hole interior belongs to the entanglement wedge of the radiation. The generalized entropy is saturated now and asymptotically becomes a constant, in which the leading order is the Bekenstein-Hawking entropy. Therefore, we reproduce the Page curve as shown in Fig. 3. 


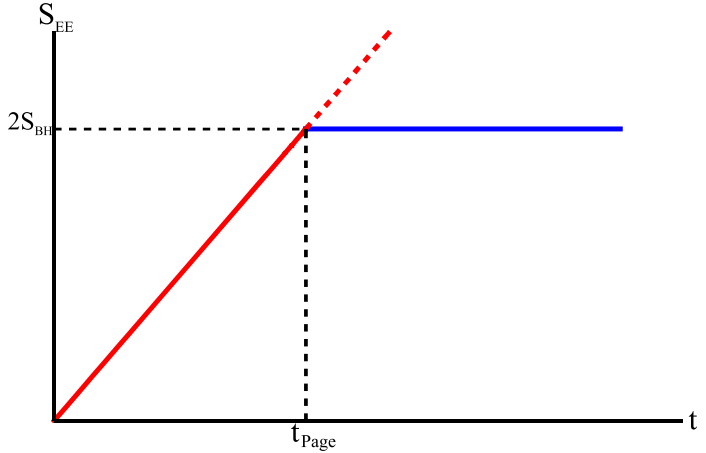

Fig. 3 The page curve for eternal GHS black holes. The red dash line represents the entropy without island while the blue solid line stands the saturation value of the entanglement entropy by considering the island

\section{Page time and scrambling time}

In this section, we give the Page time and the scrambling time, then discuss the influence of the stringy coefficient $n$ and charge $Q$ on these results. The Page time is defined as the moment when the radiation entropy of the entire system reaches maximum. For an evaporating black hole, the entropy is decreases after the Page time. But for an eternal black hole, its entropy does not change after the Page time. The relationship between the lifetime of black holes and Page time is discussed in $[9,19]$. The Page time of eternal GHS black holes can be derived from the expression (3.11) and (4.17) of entropy for two constructions. Let them be approximately equal at late times. Accordingly, the Page time as follows

$t_{\text {Page }}=\frac{6 S_{B H}}{c \kappa_{+}}=\frac{3 S_{B H}}{c \pi T_{+}}$,

where $T_{+}$is the Hawking temperature (2.11). In fact, the Page time is comparable to the half life time of evaporating black holes.

The scrambling time is defined as the recovery time of the information that fell into the black hole retrieved from the Hawking radiation [44]. In the entanglement wedge reconstruction prescription [3], the scrambling time corresponds to the time when information reaches the island, since the entanglement wedge of the island region is a part of the entanglement wedge of the radiation. At time $t=0$, if we send a message from the cut-off surface $b_{+}$, it arrives at the boundary of the island $a_{+}$at time $t^{\prime}$. The scrambling time is denoted as

$$
\begin{aligned}
t_{\mathrm{scr}} & =t^{\prime}-t=r^{*}(b)-r^{*}(a) \\
& =\left(\frac{r_{+}}{r_{+}-r_{-}}\right)^{n}\left(b-a+r_{+} \log \frac{b-r_{+}}{a-r_{+}}\right) .
\end{aligned}
$$

Substituting the location of the island (4.16), the expression of the scrambling time can be written as

$$
\begin{gathered}
t_{\mathrm{scr}} \simeq 2 r_{+}\left(\frac{r_{+}}{r_{+}-r_{-}}\right)^{n} \log \frac{r_{+}^{2}}{c G_{N}} \\
\simeq \frac{\beta}{2 \pi} \log S_{B H} \sim \beta \log \frac{t_{\text {Page }}}{\beta},
\end{gathered}
$$

where $\beta$ is the inverse temperature (2.11). The result is consistent with [45], which is very small compare to the Page time. Notice that in the second approximately equal sign, we use $\frac{r_{+}^{2}}{G_{N}} \simeq S_{B H}$. This approximation is only valid in the nonextremal case. However, for the extremal case $r_{+}=r_{-}$, the expression (5.3) is infinite, because the Bekenstein-Hawking entropy of black holes is vanishing.

We first focus on the Page time. The explicit expression for the string coefficient $n$ and the charge $Q$ as

$$
\begin{gathered}
t_{\text {Page }}(n, Q)=\frac{12 \pi}{c G_{N}} r_{+}^{3}\left(1-\frac{r_{-}}{r_{+}}\right)^{1-2 n} \\
\sim\left[1+\sqrt{\left.1-\frac{2 n}{n+1}\left(\frac{Q}{M}\right)^{2}\right]^{3}}\right. \\
{\left[1-\frac{1-\sqrt{1-\frac{2 n}{n+1}\left(\frac{Q}{M}\right)^{2}}}{n\left[1+\sqrt{1-\frac{2 n}{n+1}\left(\frac{Q}{M}\right)^{2}}\right]}\right]^{1-2 n} .}
\end{gathered}
$$

Firstly, fix the charge and investigate the influence of the stringy coefficient $n$ on the Page time. ${ }^{2}$ The results are shown in Fig. 4 a (set mass $M=100, G_{N}=1$ ). When $Q / M$ is relatively small, the Page time decreases first and then increases as $n$ increases, but the interval of the Page time varies with the stringy coefficient $n$ is very small (Fig. 5). However, in the limit case $n \rightarrow \frac{2 M^{2}}{Q^{2}}-1$, the Page time is divergent or vanishing. Note that, the Page time is obtained by considering the construction with island. But the island is not formed at early times. Accordingly, for the later case, the Page time should take a small value instead of vanishing at the extremal case.

Subsequently, we discuss the effect of the amount the charge on the Page time. The behavior of the Page time is plotted in Fig. 4b. The charge $Q$ has a very obvious influence on the Page time. For the non-extremal case, the Page time decreases monotonously with the increase of the charge $Q$. However, once again, in the limit case $Q \rightarrow \sqrt{\frac{2}{1+n}} M$, the Page time becomes divergent or zero, the later is also inaccurate for the above discussion.

\footnotetext{
${ }^{2}$ From the (2.9), the maximum charge carried by GHS black holes is $Q=\sqrt{2} M$.
} 


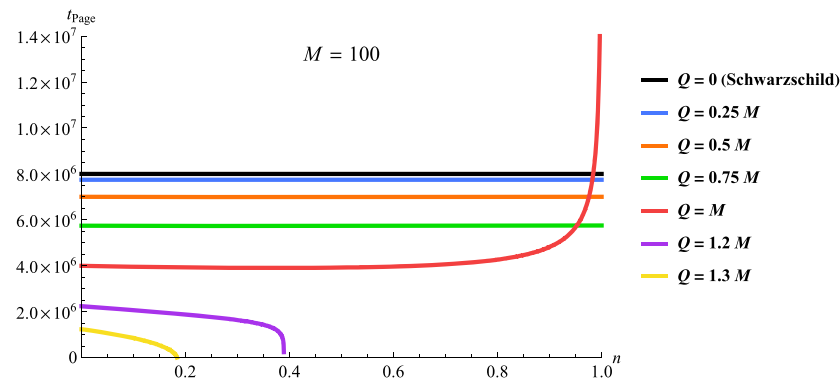

(a)

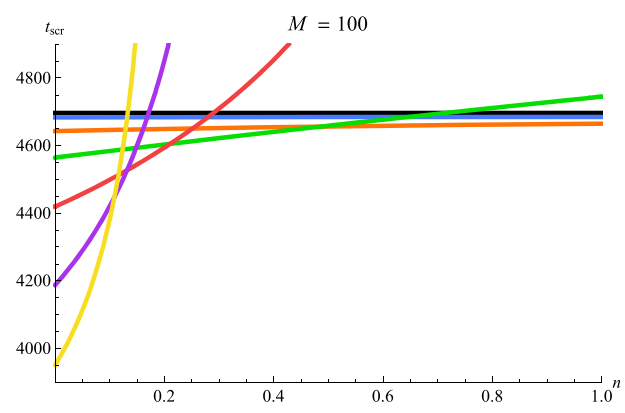

(c)

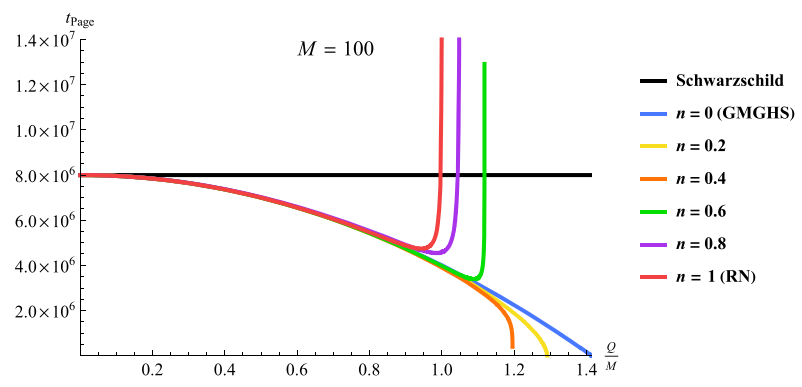

(b)

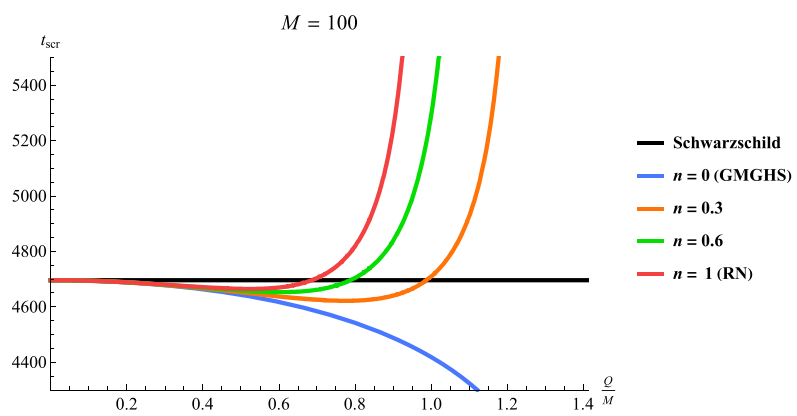

(d)

Fig. 4 The Page time and the scrambling time by GHS black holes as a function for the stringy coefficient $n$ and charge $Q$ (in the unit of $12 \pi / c$ )

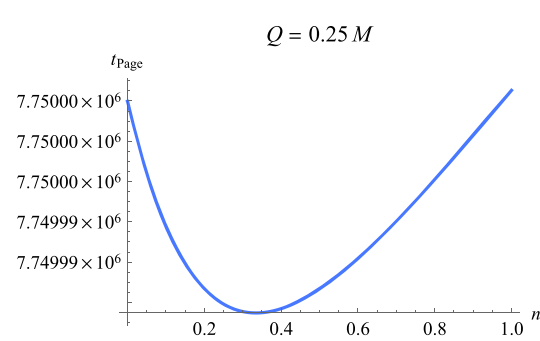

(a)

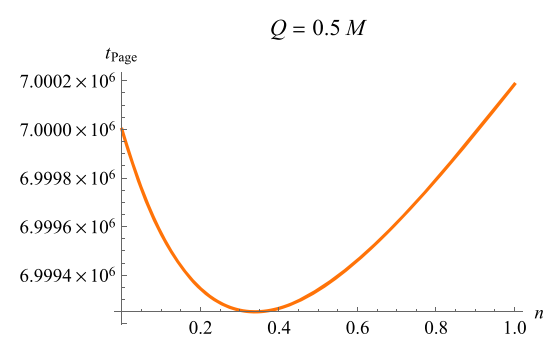

(b)

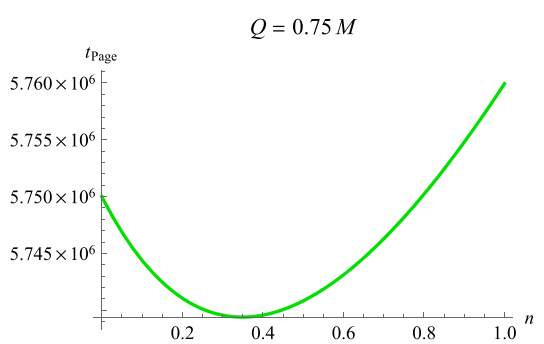

(c)

Fig. 5 The zoomed plots for Fig. 4a. The interval of the Page time varies with the stringy coefficient $n$ is very small when the charge $Q$ is small relative to the Mass $M$

Now, we consider the scrambling time. The explicit expression can be written as follows

$$
\begin{aligned}
& t_{\mathrm{scr}}(n, Q)=2 r_{+}\left(\frac{r_{+}}{r_{+}-r_{-}}\right)^{n} \log \left[\frac{\pi r_{+}^{2}}{G_{N}}\left(1-\frac{r_{-}}{r_{+}}\right)^{1-n}\right] \\
& \sim\left[1+\sqrt{1-\frac{2 n}{n+1} \frac{Q^{2}}{M^{2}}}\right]\left[1-\frac{1-\sqrt{1-\frac{2 n}{n+1} \frac{Q^{2}}{M^{2}}}}{n+n \sqrt{1-\frac{2 n}{n+1} \frac{Q^{2}}{M^{2}}}}\right]^{-n} \\
& \times \log \left(\left[1+\sqrt{1-\frac{2 n}{n+1} \frac{Q^{2}}{M^{2}}}\right]^{2}\right. \\
& \left.\left[1-\frac{1-\sqrt{1-\frac{2 n}{n+1} \frac{Q^{2}}{M^{2}}}}{n+n \sqrt{1-\frac{2 n}{n+1} \frac{Q^{2}}{M^{2}}}}\right]^{1-n}\right) .
\end{aligned}
$$

Similarly, the scrambling time changes with respect to the stringy coefficient $n$ and the charge $Q$ is shown in Fig. $4 \mathrm{c}$ and Fig. 4d respectively. As the stringy coefficient $n$ increases, the scrambling time increases monotonically in the non-extremal case. But overall, the coefficient $n$ has little impact on the result (Fig. 6). This is similar to the Page time. However, as the charge more and more large, the above expression becomes less and less accurate, since the approximation in (5.3) is only used in non-extremal case. In particular, the scrambling time is also divergent in the extremal case $\left(S_{B H}=0\right)$.

To sum up, for the Page time, in the non-extremal case, as the stringy coefficient $n$ increases, the Page time first decreases and then increases, but the overall change is so small that it can be ignored. The charge $Q$ has significant 


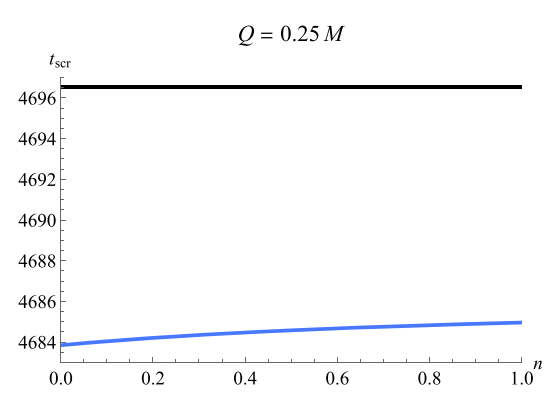

(a)

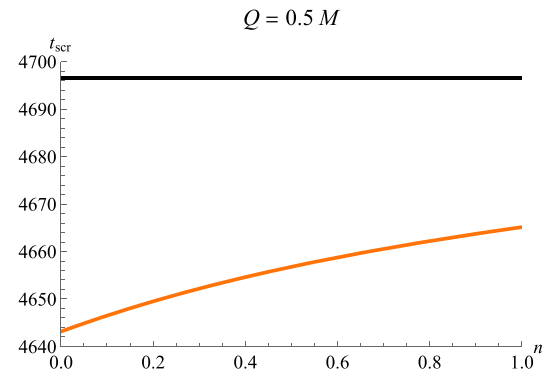

(b)

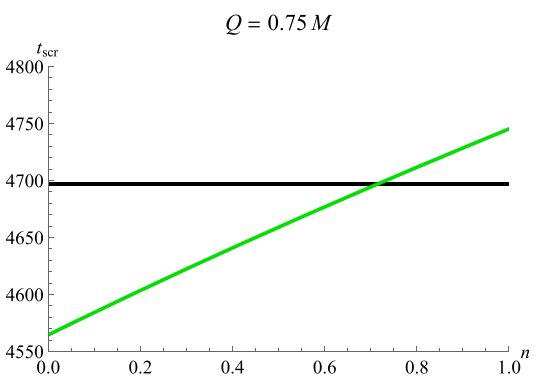

(c)

Fig. 6 The zoomed plots for Fig. 4c. The black line represents the scrambling time of Schwarzschild black holes

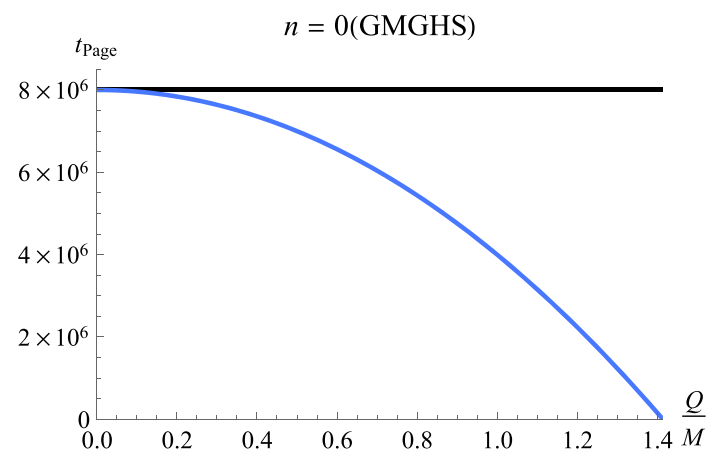

(a)

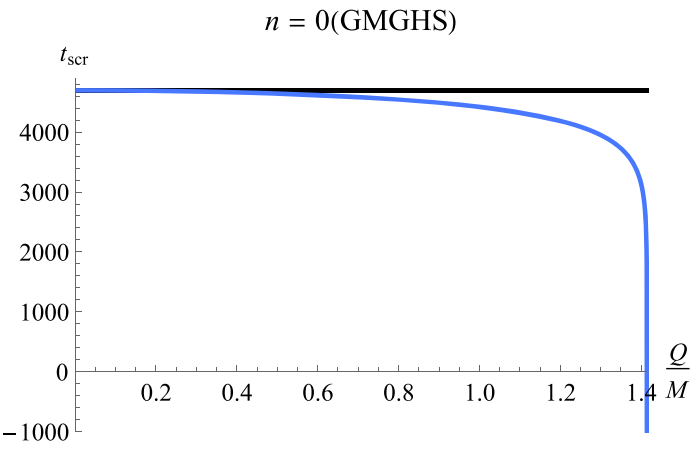

(c)

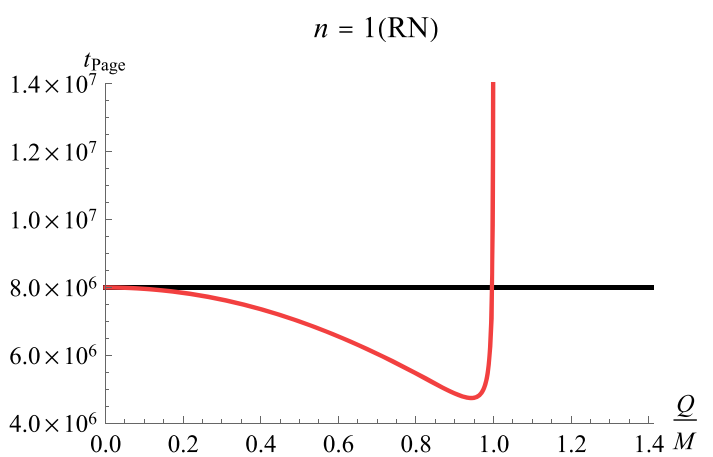

(b)

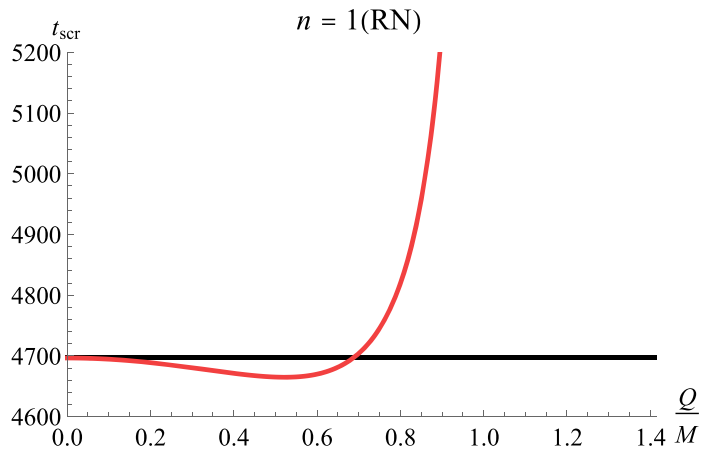

(d)

Fig. 7 The Page time and the scrambling time by GMGHS and RN black holes as a function of the charge $Q$. Black lines represents the results of Schwarzschild black holes

effect on it. The Page time decreases monotonically with charge $Q$ increases. In the extremal case, the Page time becomes divergent or near zero. For the scrambling time, although it is very small compared to the Page time, its behavior with $n$ and $Q$ is similar to the Page time. In the non-extremal case, the stringy coefficient $n$ has weak impact on it, but the charge $Q$ has a relatively strong impact on it. In the extremal case, the scrambling time is also approaches infinite. Accordingly, we should be especially careful when discussing them in near-extremal case. In addition, the Page time and the scrambling time for $n=0$ (GMGHS black holes) and $n=1$ (RN black holes) change with the charge $Q$ are plotted in Fig. 7.

\section{Conclusion and discussion}

In this paper, we study the black hole information issue in case of eternal black holes. For this system, suppose that it in the pure state at $t=0$, in the end of stage of evaporation, the amount of Hawking radiation tends to be infinite, which can produce infinite radiation entropy. Accordingly, 
Table 1 The results of the Page time and the scrambling time for different conditions. The results of Schwarzschild black holes and RN black holes are consistent with $[19,20]$

\begin{tabular}{llll}
\hline Black hole & Requirement & The Page time $t_{\text {Page }}$ & The scrambling time $t_{\text {scr }}$ \\
\hline Non-extremal GMGHS & $n=0, Q<\sqrt{2} M$ & $\frac{12 \pi}{c G_{N}} r_{+}^{3}\left(1-\frac{r_{-}}{r_{+}}\right)$ & $2 r_{+} \log \frac{r_{+}\left(r_{+}-r_{-}\right)}{G_{N}}$ \\
Extremal GMGHS & $n=0, Q=\sqrt{2} M$ & Near zero & Negative infinity \\
Non-extremal GHS & $n \in(0,1), Q<\sqrt{\frac{2}{1+n}} M$ & $\frac{12 \pi}{c G_{N}} r_{+}^{3}\left(1-\frac{r_{-}}{r_{+}}\right)^{1-2 n}$ & $\frac{2 r_{+}^{n+1}}{\left(r_{+}-r_{-}\right)^{n}} \log \frac{r_{+}^{2}}{G_{N}}\left(\frac{r_{+}-r_{-}}{r_{+}}\right)^{1-n}$ \\
Extremal GHS & $n \in(0,1), Q=\sqrt{\frac{2}{1+n}} M$ & Near zero/divergent & Divergent \\
Non-extremal RN & $n=1, Q<M$ & $\frac{12 \pi}{c G_{N}} r_{+}^{3}\left(1-\frac{r_{-}}{r_{+}}\right)^{-1}$ & $\frac{2 r_{+}^{2}}{r_{+}-r_{-}} \log \frac{r_{+}^{2}}{G_{N}}$ \\
Extremal RN & $n=1, Q=M$ & Divergent & Divergent \\
Schwarzschild & $Q=0$ & $\frac{12 \pi}{c G_{N}} r_{+}^{3}$ & $2 r_{+} \log \frac{r_{+}^{2}}{G_{N}}$
\end{tabular}

the result violates the Bekenstein entropy bound and also conflicts with the unitarity of quantum mechanics. Therefore, we can present a version of the information paradox.

Based on a series of important solutions of the Einstein's equation in low-energy string theory, we study the information paradox in four dimensional GHS spacetime. At early times, no island is formed. The entanglement entropy of the system is contributed from matter fields (3.1). Therefore, the radiation entropy keeps increasing linearly with time (3.11). However, at late times, the island emerges outside the event horizon, which changes the construction of the system. Now the entanglement wedge of the black hole interior belongs to the entanglement wedge of the radiation. The entanglement entropy is dominated by the contribution from the island region (4.17). The entire behavior of the generalized entropy can be described from the Page curve in the Fig. 3. For these results, we obtain the Page time (5.1) and the scrambling time (5.3) and discuss the impact of stringy coefficient $n$ and charge $Q$ on them. All data are shown in Table 1. In particular, we should pay attention to the extremal black hole. The Page time and the scrambling time become divergent or near vanishing, which requires further investigation on the island formula (1.1).

In the future, we should also pay attention to the following issues:

(1) The paper only studies the situation of a single island. Related work indicates that the construction of multiple islands may produce additional entanglement entropy, but their contribution is higher order and can be ignored. These islands probably appear at near the Page time, thus alleviating the phase transition of entanglement entropy [19].

(2) The background of the paper is the asymptotically flat spacetime. However, the Page curve is obtained first in the asymptotically AdS spacetime, where the bath is coupled to collect the radiation. Therefore, one should be careful to investigate the island in the asymptotically flat spacetime.
(3) The calculation in this paper is done by simply using the two-dimensional approximation formula, but the $s$-wave reduction of the entanglement entropy still need further justification in higher dimensional spacetime. We expect that there is a better calculation to obtain the entanglement entropy in higher dimensional spacetime, which can verify the island formula (1.1).

(4) It is worth noting that in the extremal case, the behaviors of both the Page time and the scrambling time lacks a reasonable explanation. This also implies that the island formula needs more verification.

(5) At last, from the aspect of information theory, the Page curve is obtained by considering the construction of the island. But how the information that trapped in the island is connected to the outside of the radiation. An innovative conjecture is " $E R=E P R$ " [46]. However, this conjecture still lacks a strong proof. Accordingly, we also expect to extracting the information residing in islands by theoretically feasible protocol.

In all, we still need a complete theory of quantum gravity to explain the microscopic mechanism of black hole evaporation. The black hole information issue requires further study on the island paradigm.

\section{Note added}

After we have finished this paper, we receive a very interesting paper [30], where the authors introduce an alternative method on taking extremal limit of black holes. It would be interesting to perform a similar analysis on our black hole background.

Acknowledgements We would like to thank Yang Zhou, Chen-Yang Dong, Cheng-Yuan Lu and Yu-Qi Lei for helpful discussions. The study was partially supported by NSFC, China (Grant no. 11875184). 
Data Availability Statement This manuscript has no associated data or the data will not be deposited. [Authors' comment: There are no associated data available.]

Open Access This article is licensed under a Creative Commons Attribution 4.0 International License, which permits use, sharing, adaptation, distribution and reproduction in any medium or format, as long as you give appropriate credit to the original author(s) and the source, provide a link to the Creative Commons licence, and indicate if changes were made. The images or other third party material in this article are included in the article's Creative Commons licence, unless indicated otherwise in a credit line to the material. If material is not included in the article's Creative Commons licence and your intended use is not permitted by statutory regulation or exceeds the permitted use, you will need to obtain permission directly from the copyright holder. To view a copy of this licence, visit http://creativecomm ons.org/licenses/by/4.0/.

Funded by SCOAP ${ }^{3}$.

\section{References}

1. S.W. Hawking, Breakdown of predictability in gravitational collapse. Phys. Rev. D 14, 2460-2473 (1976)

2. A. Almheiri, T. Hartman, J. Maldacena, E. Shaghoulian, A. Tajdini, Replica wormholes and the entropy of Hawking radiation. JHEP 05, 013 (2020). arXiv:1911.12333 [hep-th]

3. G. Penington, Entanglement wedge reconstruction and the information paradox. JHEP 09, 002 (2020). arXiv:1905.08255 [hep-th]

4. A. Almheiri, R. Mahajan, J. Maldacena, Y. Zhao, The Page curve of Hawking radiation from semiclassical geometry. JHEP 03, 149 (2020). arXiv:1908.10996 [hep-th]

5. A. Almheiri, T. Hartman, J. Maldacena, E. Shaghoulian, A. Tajdini, The entropy of Hawking radiation. Rev. Mod. Phys. 93, 35002 (2021). arXiv:2006.06872 [hep-th]

6. A. Almheiri, N. Engelhardt, D. Marolf, H. Maxfield, The entropy of bulk quantum fields and the entanglement wedge of an evaporating black hole. JHEP 12, 063 (2019). arXiv:1905.08762 [hep-th]

7. S.W. Hawking, Particle creation by black holes. Commun. Math. Phys. 43, 199 (1975) [Erratum: Commun. Math. Phys. 46, 206 (1976)]

8. D.N. Page, Information in black hole radiation. Phys. Rev. Lett. 71, 3743-3746 (1993). arXiv:hep-th/9306083

9. D.N. Page, Time dependence of Hawking radiation entropy. JCAP 1309, 028 (2013). arXiv:1301.4995 [hep-th]

10. J. Maldacena, The large $\mathrm{N}$ limit of superconformal field theories and supergravity. Int. J. Theor. Phys. 38, 1113-1133 (1999). arXiv:hep-th/9711200

11. A. Almheiri, D. Marolf, J. Polchinski, J. Sully, Black holes: complementarity or firewalls? JHEP 02, 062 (2013). arXiv:1207.3123 [hep-th]

12. J.D. Bekenstein, A universal upper bound on the entropy to energy ratio for bounded systems. Phys. Rev. D 23, 287 (1981)

13. A. Almheiri, R. Mahajan, J. Maldacena, Islands outside the horizon. arXiv:1910.11077 [hep-th]

14. H.Z. Chen, Z. Fisher, J. Hernandez, R.C. Myers, S.M. Ruan, Information flow in black hole evaporation. JHEP 2003, 152 (2020). arXiv:1911.03402 [hep-th]

15. T.J. Hollowood, S.P. Kumar, Islands and Page curves for evaporating black holes in JT gravity. JHEP 08, 094 (2020). arXiv:2004.14944 [hep-th]

16. T. Anegawa, N. Iizuka, Notes on islands in asymptotically flat $2 \mathrm{~d}$ dilaton black holes. JHEP 07, 036 (2020). arXiv:2004.01601 [hepth]
17. F.F. Gautason, L. Schneiderbauer, W. Sybesma, L. Thorlacius, Page curve for an evaporating black hole. JHEP 05, 091 (2020). arXiv:2004.00598 [hep-th]

18. T. Hartman, E. Shaghoulian, A. Strominger, Islands in asymptotically flat 2D gravity. JHEP 07, 022 (2020). arXiv:2004.13857 [hepth]

19. K. Hashimoto, N. Iizuka, Y. Matsuo, Islands in Schwarzschild black holes. JHEP 06, 085 (2020). arXiv:2004.05863 [hep-th]

20. X. Wang, R. Li, J. Wang, Islands and Page curves of ReissnerNordström black holes. JHEP 04, 103 (2021). arXiv:2101.06867 [hep-th]

21. W. Kim, M. Nam, Entanglement entropy of asymptotically flat nonextremal and extremal black holes with an island. Eur. Phys. J. C 81, 869 (2021). arXiv:2103.16163 [hep-th]

22. Y. Ling, Y. Liu, Z.Y. Xian, Island in charged black holes. JHEP 03, 251 (2021). arXiv:2010.00037 [hep-th]

23. G.K. Karananas, A. Kehagias, J. Taskas, Islands in linear dilaton black holes. JHEP 03, 253 (2021). arXiv:2101.00024 [hep-th]

24. A. Almheiri, R. Mahajan, J.E. Santos, Entanglement islands in higher dimensions. SciPost Phys. 9(1), 001 (2020). arXiv:1911.09666 [hep-th]

25. H. Geng, A. Karch, Massive islands. JHEP 09, 121 (2020). arXiv:2006.02438 [hep-th]

26. X. Wang, R. Li, J. Wang, Islands and Page curves for a family of exactly solvable evaporating black holes. Phys.Rev. D 103, 126026 (2021). arXiv:2104.00224 [hep-th]

27. J. Chu, F. Deng, Y. Zhou, Page curve from defect extremal surface and island in higher dimensions. JHEP 10, 149 (2021). arXiv:2105.09106 [hep-th]

28. E. Caceres, A. Kundu, A. Patra, S. Shashi, Page curves and bath deformations. arXiv:2107.00022 [hep-th]

29. C. Krishnan, V. Patil, J. Pereira, Page curve and the information paradox in flat space. arXiv:2005.02993 [hep-th]

30. B. Ahn, S. Bak, H. Jeong, K. Kim, Y. Sun, Islands in charged linear dilaton black holes. arXiv:2107.07444] [hep-th]

31. H. Geng, A. Karch, C.P. Pardavila, S. Raju, L. Randall, M. Riojas, S. Shashi, Inconsistency of islands in theories with long-range gravity. arXiv:2107.03390 [hep-th]

32. S. Ryu, T. Takayanagi, Holographic derivation of entanglement entropy from AdS/CFT. Phys. Rev. Lett. 96, 181602 (2006). arXiv:hep-th/0603001

33. A. Lewkowycz, J. Maldacena, Generalized gravitational entropy. JHEP 08, 090 (2013). arXiv:1304.4926 [hep-th]

34. T. Barrella, X. Dong, S.A. Hartnoll, V.L. Martin, Holographic entanglement beyond classical gravity. JHEP 09, 109 (2013). arXiv:1306.4682 [hep-th]

35. T. Faulkner, A. Lewkowycz, J. Maldacena, Quantum corrections to holographic entanglement entropy. JHEP 11, 074 (2013). arXiv:1307.2892 [hep-th]

36. N. Engelhardt, A.C. Wall, Quantum extremal surfaces: holographic entanglement entropy beyond the classical regime. JHEP 01, 073 (2015). arXiv:1408.3203 [hep-th]

37. C. Akers, N. Engelhardt, G. Penington, M. Usatyuk, Quantum maximin surfaces. arXiv:1912.02799 [hep-th]

38. A.C. Wall, Maximin surfaces, and the strong subadditivity of the covariant holographic entanglement entropy. Class. Quantum Gravity 31(22), 225007 (2014). arXiv:1211.3494 [hep-th]

39. G. Penington, S.H. Shenker, D. Stanford, Z. Yang, Replica wormholes and the black hole interior. arXiv:1911.11977 [hep-th]

40. D. Garfinkle, G.T. Horowitz, A. Strominger, Charged black holes in string theory. Phys. Rev. D 43, 3140 (1991)

41. G.W. Gibbons, K. Maeda, Black holes and membranes in higherdimensional theories with dilaton fields. Nucl. Phys. B 298, 741 (1988) 
42. T.M. Fiola, J. Preskill, A. Strominger, S.P. Trivedi, Black hole thermodynamics and information loss in two-dimensions. Phys. Rev. D 50, 3987-4014 (1994). arXiv:hep-th/9403137

43. H. Casini, C.D. Fosco, M. Huerta, Entanglement and alpha entropies for a massive Dirac field in two dimensions. J. Stat. Mech. 0507, P07007 (2005). arXiv:cond-mat/0505563

44. P. Hayden, J. Preskill, Black holes as mirrors: quantum information in random subsystems. JHEP 09, 120 (2007). arXiv:0708.4025 [hep-th]
45. Y. Sekino, L. Susskind, Fast scramblers. JHEP 10, 065 (2008). arXiv:0808.2096 [hep-th]

46. J. Maldacena, L. Susskind, Cool horizons for entangled black holes. Fortschr. Phys. 61, 781-811 (2013). arXiv:1306.0533 [hep-th] 